\title{
Sentencia del Tribunal Constitucional recaída en control preventivo y obligatorio de constitucionalidad, del proyecto de ley que establece el Sistema de Elecciones Primarias para la nominación de candidatos a Presidente de la República, Senadores, Diputados y Alcaldes.
}

\section{STC Rol No 2324-2012, de 20 de noviembre de 2012}

\section{Doctrina:}

- La potestad normativa entregada al Servicio Electoral excede los límites que resultan constitucionalmente aceptables, dado que el sistema de elecciones primarias debía ser regulado por una ley orgánica constitucional, que al menos debe definir aspectos jurídicos esenciales.

- Son inconstitucionales las disposiciones que contradicen el principio de legalidad en materia penal, al hacer referencia indeterminada a tipos penales, sin especificar suficientemente la conducta penada.

\section{Requerimiento:}

- El control de constitucionalidad de las normas del proyecto de ley se realiza en virtud del Art. 93 inciso primero No 1 de la Constitución Política de la República (CPR), que otorga al Tribunal Constitucional la facultad, entre otras, de ejercer el control abstracto, preventivo y obligatorio de las leyes orgánicas constitucionales.

- El procedimiento constitucional se inicia por oficio de la Cámara de Diputados, que remite el proyecto de ley que establece el sistema de elecciones primarias para la nominación de candidatos a Presidente de la República, senadores, diputados y alcaldes, para que se realice el control de constitucionalidad de la totalidad del proyecto.

* Profesor Titular de Derecho Constitucional, Facultad de Derecho, Universidad de Chile. 


\section{Ley Orgánica Constitucional, principio de competencia:}

Como hemos dicho, la Cámara de Diputados remite el proyecto de ley, a fin de que realice el control de constitucionalidad de todo el proyecto. Luego de reproducir el tenor del mismo, la sentencia fija su competencia respecto de las materias que resulten propias del dominio de ley orgánica constitucional en el proyecto, señala la fuente constitucional respectiva, de acuerdo a lo siguiente:

OCTAVO.- Que, conforme con la interpretación que deriva de su texto, con la naturaleza de las leyes orgánicas constitucionales dentro de nuestra normativa jurídica y con el espiritu del constituyente al incorporarlas a nuestra Carta Fundamental, las disposiciones del proyecto consultadas que se indicarán en los considerandos siguientes de esta sentencia están comprendidas, según corresponda, dentro de las materias que el Poder Constituyente ha encomendado que sean reguladas por las leyes orgánicas constitucionales a que se refieren los incisos cuarto a sexto de esta sentencia;

NOVENO.- Que las disposiciones contenidas en los artículos $1^{\circ}$ a $47, y$ primero transitorio del proyecto de ley sometido a control de constitucionalidad, son propias de las leyes orgánicas constitucionales sobre Sistema Electoral Público; sobre Sistema de financiamiento, transparencia, limite y control del gasto electoral, y sobre Sistema de Registro Electoral a que se refiere el artículo 18 de la Constitución Politica; y de la Ley Orgánica Constitucional sobre Sistema de elecciones primarias que podrá ser utilizado por los partidos politicos para la nominación de candidatos a cargos de elección popular, a que se refiere el artículo $19, N^{\circ} 15$, inciso quinto, de la Constitución Politica;

DÉCIMO.- Que las disposiciones contenidas en los artículos 32 a 36 del proyecto de ley remitido, son, asimismo, propias de la Ley Orgánica Constitucional sobre el Tribunal Calificador de Elecciones a que se refiere el inciso primero del artículo 95 de la Ley Fundamental;"

\section{Potestad normativa del SERVEL:}

La sentencia declara inconstitucional el Art. 25 del proyecto, que señala que el Servicio Electoral regulará diversas materias relacionadas con la elección, lo que a juicio del Tribunal vulnera la reserva de ley, en cuanto a la regulación de las elecciones primarias.

La potestad entregada al Servicio Electoral es del siguiente tenor:

"DECIMOCUARTO.- Que, como se observa, la norma entrega una potestad normativa al Servicio Electoral para regular ciertos aspectos de la elección.

Dichos aspectos son definidos de dos maneras. Por una parte, de manera especifica. La normativa que debe dictar el Servicio Electoral se refiere a "la propaganda electoral", "la votación" y "el escrutinio". Por la otra, de manera genérica, el proyecto señala que le corresponde al Servicio Electoral dictar normas sobre, "en general, todo aquello relativo al acto eleccionario"; 
Luego, la sentencia hace una referencia al contenido de la potestad normativa, y al hecho de que no hay un reproche de constitucionalidad a la regulación que la otorgue de forma general, pero que se deben cumplir con ciertas condiciones:

"DECIMOQUINTO.- Que este Tribunal Constitucional no encuentra reproche en que se entregue potestad normativa a un servicio público. Diversas normas de la Constitución se refieren a esta posibilidad. Por de pronto, el articulo 65, inciso cuarto, $N^{\circ} 2$, establece que es materia de ley, de iniciativa exclusiva del Ejecutivo, determinar "las atribuciones de los servicios públicos". Entre esas facultades puede estar la de dictar normas. La Constitución faculta, entonces, a la ley para conferir esta potestad a dicho servicio. La potestad normativa, por tanto, requiere texto expreso. No es inherente a la jefatura, como puede ser la de dictar instrucciones. Enseguida, la Constitución se refiere a esta potestad normativa cuando regula la toma de razón. Esta recae en decretos y resoluciones (artículo 99). Estas son las órdenes escritas, sobre asuntos de su competencia, que dictan las autoridades dotadas de poder de decisión (artículo $3^{\circ}$, inciso quinto, Ley $N^{\circ} 19.880$ ). Finalmente, la Constitución se refiere a la potestad normativa de órganos distintos al Presidente de la República cuando regula la potestad normativa de los municipios (artículo 119, inciso segundo) y de los gobiernos regionales (articulo 113);

DECIMOSEXTO.- Que, como toda potestad normativa secundaria, debe sujetarse a la Constitución, a la ley y a la normativa reglamentaria que dicte el Presidente de la República. También sólo puede pormenorizar o detallar, sin innovar, lo establecido en la ley;

DECIMOSÉPTIMO.- Que tampoco considera esta Magistratura que haya inconveniente en que exista esta potestad normativa para pormenorizar o detallar asuntos que están regulados en una ley orgánica. Estas leyes, si bien deben abordar ciertas materias y, en tal sentido, se rigen por el principio de competencia, y están sujetas a una superlegalidad de forma, por requerir un quórum más alto de aprobación que las leyes comunes, y estar sujetas al control preventivo de constitucionalidad de esta Magistratura, no dejan de ser leyes, pues emanan de la potestad legislativa. En tal sentido, pueden requerir de pormenorización o detalle a cargo de una normativa secundaria;"

Finalmente, en este punto, la sentencia concluye que, en este caso, la potestad normativa entregada al Servicio Electoral excede los límites que resultan constitucionalmente aceptables, dado que el sistema de elecciones primarias debía ser regulado por una ley orgánica constitucional, que al menos debe definir aspectos jurídicos esenciales. Al respecto señala el Tribunal:

"DECIMONOVENO.- Que, sin embargo, esta potestad normativa conferida al Servicio Electoral se da en un contexto especial, dada la forma en que el proyecto definió el marco jurídico que rige a las elecciones primarias, en el artículo $6^{\circ}$. Ahi se remitió "en todo lo que no sea contrario a ella y en lo que le sea aplicable", a las leyes $N^{\circ}$ s 18.700, 18.556, 18.695 y 18.603. Por lo mismo, deja un amplio margen para que dicha potestad normativa pueda definir lo que sea aplicable de ellas; 
VIGÉSIMO.- Que, en este sentido, tal potestad normativa, que permite que se pueda regular por el Servicio Electoral, entre otras materias, "en general todo aquello relativo al acto eleccionario", vulnera la Constitución;

VIGESIMOPRIMERO.-Que tal vulneración se produce, en primer lugar, porque la Constitución manda que sea "una ley orgánica constitucional" la que establezca "un sistema de elecciones primarias”. En tal sentido, el legislador orgánico debe precisar qué normas son las que regulan estas elecciones. Es la ley orgánica la que debe establecer el régimen jurídico de las primarias, no dejando en manos del Servicio Electoral definir ese marco jurídico en aspectos esenciales. El legislador puede establecer ese todo armónico y sistemático directamente o remitiendo a otras disposiciones legales. Pero no puede confiar esa tarea a la autoridad administrativa. En segundo lugar, tal potestad deslegaliza un ámbito que la Constitución reservó a la competencia del legislador orgánico. Cuando la Constitución encarga a la ley orgánica regular algo, el legislador debe hacerlo, sin que quepa que la potestad normativa secundaria pueda llenar ese vacio, pues ésta es de pormenorización o detalle de un contenido que la ley, en lo medular, ya reguló. Recordemos que ni siquiera el decreto con fuerza de ley cabe en materias propias de ley orgánica constitucional (artículo 64 de la Ley Suprema). Menos puede hacerlo una normativa que no es ni siquiera de rango legal;"

Por lo anterior, se declara como inconstitucional el Art. 25 del proyecto, en la parte en que entrega al Servicio Electoral la facultad de dictar una serie de normas relacionadas con el desarrollo del proceso electoral.

\section{Identidad del Ciudadano Elector:}

A continuación, se declara inconstitucional el Art. 29 del proyecto, el cual excluye la aplicación del Art. 63 de la Ley No 18.700, relativo a la identificación de electores.

Al efecto, la sentencia señala:

"VIGESIMOCUARTO.- Que el artículo 63 citado, en lo pertinente, dispone que:

"Si a juicio de la mesa existiere disconformidad notoria y manifiesta entre las indicaciones del padrón de mesa y la identidad del elector, se recabará la intervención del experto de identificación que habrá en cada local de votación. El experto hará que el elector estampe su huella dactilar derecha al lado de su firma y la cotejará con la estampa en su cédula nacional de identidad o cédula de identidad para extranjeros".

Adicionalmente, dicho precepto dispone la modalidad en que este problema debe ser resuelto: VIGESIMOQUINTO.- Que consideramos que dicha exclusión es inconstitucional, en primer lugar, porque no es razonable que el peso de la organización electoral en la mesa de sufragio resida en ciudadanos en el ejercicio de una carga pública (los vocales) y, al 
mismo tiempo, se exonere a un organismo del Estado de un deber crucial de identificación del elector;"

Además, se estima que la norma considerada inconstitucional establece una exclusión que podría afectar los derechos de las personas:

"VIGESIMOCTAVO.- Que, en sintesis, consideramos que la norma establece una exclusión que puede afectar el ejercicio de derechos politicos inherentes a la condición de ciudadano, establecidos en la Constitución;"

\section{Principio de legalidad penal:}

En tercer lugar, la sentencia declara la inconstitucionalidad de los artículos 44 y 45 del proyecto, por estimar que contradicen el principio de legalidad en materia penal, al hacer una referencia indeterminada a tipos penales, sin especificar suficientemente la conducta penada.

La remisión se da en los siguientes términos:

"TRIGÉSIMO.-Que, como se observa, dichos preceptos regulan las sanciones que pueden aplicarse durante el proceso eleccionario de las elecciones primarias y las sanciones que puedan aplicarse a los partidos políticos.

Pero lo hacen de una manera especial, porque remiten a lo señalado en la Ley $N^{\circ}$ 18.700, en los artículos 124 a $153 \mathrm{~A}$, o a lo establecido en los Títulos VIII y IX de la Ley $N^{\circ}$ 18.603. En estas disposiciones se establecen faltas y delitos y el procedimiento para perseguir la responsabilidad consiguiente.

Sin embargo, esa remisión no es integra, sino que es "en lo que fuere procedente" o "en lo que corresponda";

Luego se analiza la amplitud de la remisión y la determinación de la conducta punible, para concluir que no resultan lícitos los artículos del proyecto en cuestión.

"TRIGESIMOSEGUNDO.- Que, sin embargo, si le compete examinar si esa remisión es lícita.

En efecto, si bien es posible que una ley remita a otra ley para los efectos de definir la conducta probibida y su sanción (leyes penales en blanco impropias), no es lícito constitucionalmente que no se precise con claridad cuáles son las conductas lícitas y las prohibidas de esa remisión.

Esto es lo que ocurre en la remisión que se hace al Titulo VII de la Ley $N^{\circ} 18.700$, al utilizar la expresión "en lo que fuere procedente". Y lo que se hace con la remisión a los Titulos VIII y IX de la Ley $N^{\circ} 18.603$, "en lo que corresponda";

TRIGESIMOTERCERO.- Que los destinatarios de una norma que establece sanciones por faltas y delitos, deben saber con antelación y con seguridad juridica qué conductas se les pueden reprochar si las llegan a cometer; deben poder obtener del texto normativo la representación cabal de la conducta. La tipicidad exige el conocimiento anticipado por las 
personas del comportamiento que la ley sanciona. La legalidad de la sanción y la tipicidad son garantías que no pueden obviarse, ni aun en este tipo de leyes. Más todavía si entre las disposiciones remitidas hay algunas que configuran delitos. La función garantista de una ley cierta y expresa, en que la conducta que se sanciona esté no solamente descrita sino que pueda ser identificada con total claridad, se vería seriamente afectada si las personas no saben con precisión la conducta punible.

Las normas contenidas en los artículos 44 y 45 del proyecto de ley bajo análisis tampoco cumplen con el requisito constitucional tantas veces declarado por este Tribunal Constitucional en el sentido de que todo tipo penal debe establecer, a lo menos, el núcleo esencial de la conducta punible, cuestión que no acontece en la especie, toda vez que las normas referidas no determinan suficientemente dicha conducta punible, ni siquiera en sus rasgos esenciales;

TRIGESIMOCUARTO.- Que el legislador no puede realizar una remisión de la naturaleza de la contenida en la norma que se analiza, que introduce una incertidumbre, insubsanable, al disponer que se van a sancionar delitos y faltas "en lo que fuere procedente".

Si bien el legislador puede permitir que el juez desentrañe el sentido de una norma, incluso, interpretando los elementos normativos del tipo penal, no puede no establecer de manera cierta cuáles son las conductas reprochables. En tal sentido, debe precisar qué disposiciones se aplican y cuáles no de la ley remitida, sin incorporar en la norma de remisión elementos abiertos, que pueden generar confusión, ambigüedad o incertidumbre en la configuración de la conducta punible;"

\section{Votos disidentes y prevenciones:}

La sentencia es acordada, en sus diversos puntos, con muchas disidencias y prevenciones de diversos Ministros, de los cuales se puede destacar.

Los Ministros Hernán Vodanovic Shnake, José Antonio Viera-Gallo Quesney e Iván Aróstica Maldonado, estuvieron en contra de la decisión de declarar inconstitucional el Art. 25 del proyecto, que entrega facultades normativas al Servicio Electoral. Se sostiene que, a diferencia del Art. 18 inciso primero de la Constitución, que señala que la ley determinará un sistema electoral público, regulando su organización y funcionamiento, el Art. 19 № 15, que establece las elecciones primarias, solamente encomienda a la ley el establecimiento de elecciones primarias, no se regulación específica.

Señalan en este sentido los disidentes:

“4 $4^{\circ}$ Que, con menos amplitud respecto a las materias que encomienda legalizar, el mencionado artículo $19, N^{\circ} 15$, en la segunda oración del párrafo quinto, solo dispone que "Una ley orgánica constitucional establecerá un sistema de elecciones primarias que podrá ser utilizado por dichos partidos para la nominación de candidatos a cargos de elección popular, cuyos resultados serán vinculantes para estas colectividades, salvo las excepciones que establezca dicha ley. Aquellos que no resulten elegidos en las elecciones primarias no podrán ser candidatos, en esa misma elección, al respectivo cargo". 
Porque este tipo de comicios facultativos no posee igual trascendencia que aquellas elecciones periódicas que comprometen las bases de la institucionalidad $\left(\operatorname{Rol~} N^{\circ} 53\right.$, considerandos $4^{\circ}$ y $\left.5^{\circ}\right)$, es que el constituyente optó aquí por confiar al desarrollo posterior de la ley solo lo concerniente al "sistema" de elecciones primarias, vale decir, sus principios básicos y reglas generales, pero -por contraste con el citado inciso primero del artículo 18 constitucional- sin hacer extensiva esta ley a la "organización” ni a "la forma en que se realizarán" tales procesos;

$5^{\circ}$ ) Que, dentro de este amplio margen y sin una experiencia previa que fuera prudente petrificar en una ley orgánica constitucional, el articulo $3^{\circ}$ del proyecto, en el Párrafo $1^{\circ}$ sobre "la realización" de las elecciones primarias, encarga al Servicio Electoral "organizar" dichas votaciones, esto es, ordenar las actividades conducentes a su efectiva concreción dentro del aludido marco constitucional y legal.

De donde se entiende claramente que, más adelante, el artículo 25 faculte al mismo organismo para dictar las normas administrativas e instrucciones que permitan materializar estos procesos de nominación, tal como puede hacerlo actualmente el Consejo Directivo del Servicio Electoral, de conformidad con el artículo 67, letra e), de la Ley $N^{\circ}$ 18.556. Atribución ésta que, por lo demás, puede recibir plena aplicación y que no se ve menoscabada por la inconstitucionalidad con que se disiente, habida cuenta de la remisión que a dicho cuerpo legal efectúa el artículo $6^{\circ}$ del proyecto;

$\left.6^{\circ}\right)$ Que, por último, no puede sostenerse la inconstitucionalidad del artículo 25 del proyecto por ser incongruente con el artículo $6^{\circ}$ del mismo. Menos cuando se entiende que este último se remite a la Ley $N^{\circ} 18.700$, precisamente porque - de suyo-las elecciones primarias no se rigen por dicha ley, desde que sería superfluo e innecesario hacer aplicable un régimen jurídico a situaciones que por si mismas están afectas a él.

En esta lógica del proyecto, entonces, las elecciones primarias se habrian de "organizar" por el Servicio Electoral conforme a las normas de esta ley dictada en virtud del $N^{\circ} 15 \mathrm{del}$ artículo 19 constitucional (articulo $1^{\circ}$ ); a las disposiciones de la remitida Ley $N^{\circ} 18.700$, en lo compatible e inútil de repetir por razones de economía legislativa (artículo $6^{\circ}$ ), y a las reglas adoptadas por el directorio de esa institución, en los aspectos administrativos especificos para las que han sido habilitadas (artículo 25\%)."

De la misma forma, la Ministra Marisol Peña Torres y los Ministros José Antonio VieraGallo Quesney e Iván Aróstica Maldonado, estuvieron en contra de la declaración de inconstitucionalidad del Art. 29 del proyecto, sobre la identificación de los electores, que excluye la aplicación del Art-63 de la Ley No 18.700.

Los disidentes estiman que no se produce efecto inconstitucional entregando a la mesa la decisión en caso de problemas de identidad del elector, aún cuando no haya intervención de un órgano público, puesto que existen medidas para evitar una posible arbitrariedad en este tipo de decisiones.

"5". Que, sobre el particular, debe tenerse presente que la posible arbitrariedad con que pudiera actuar la Mesa Receptora de Sufragios en esta materia se ve claramente contrarrestada con las sanciones que establece, para los miembros de dichas Mesas, el articulo 132, especialmente en 
sus numerales 4) y 8), de la Ley $N^{\circ} 18.700$, expresamente aplicable en la especie por disposición del artículo 44 del proyecto de ley examinado, lo que, en concepto de quienes suscriben este voto es suficiente garantía del cabal y adecuado ejercicio del derecho de sufragio sin que se vea afectada la constitucionalidad del articulo 29 del proyecto de ley de que se trata.

$6^{\circ}$. Que, a mayor abundamiento, debe consignarse que la decisión de excluir a los expertos en las elecciones primarias ha sido tomada por los poderes colegisladores dentro del ejercicio de sus facultades constitucionales, de modo tal que, no obstante que pueda hacerse un juicio de mérito acerca de la conveniencia o inconveniencia de la opción adoptada por el legislador orgánico constitucional, ello no constituye una cuestión de constitucionalidad ni transforma al precepto en comento en contrario a la Carta Fundamental;

$7^{\circ}$. Que, por último, no está de más tener presente que el mismo legislador ha establecido en el proyecto bajo examen un procedimiento para la determinación de los padrones electorales en que intervienen los partidos politicos o pactos electorales respectivos y el Servicio Electoral, debiendo éste último configurar el padrón definitivo. Luego, por ejemplo, el proyecto dispone que el padrón contendrá sólo los nombres y apellidos del elector y su número de rol único nacional, simplificando asi el padrón y aplicando una regla especial por sobre las normas de la Ley $N^{\circ}$ 18.556, Orgánica Constitucional sobre Sistema de Inscripciones Electorales y Servicio Electoral. Ello, al igual que la exclusión de los expertos en identificación, es una decisión soberana del legislador que persigue simplificar los procesos de elecciones primarias, opción que cuestionable o no en su mérito, no acarrea efectos inconstitucionales."

Por otro lado, la Ministra Marisol Peńa Torres, y los Ministros Carlos Carmona Santander y Gonzalo García Pino, estuvieron por declarar la inconstitucionalidad del Art. 37 del proyecto, en la parte que el mismo señala que los perdedores de las elecciones primarias no podrán ser candidatos al mismo cargo, en el mismo territorio.

Se señala por los disidentes, que la Constitución fija una serie de prohibiciones para los candidatos perdedores de una elección primaria, quienes no podrán ser candidatos al mismo cargo, en la elección inmediatamente posterior a la elección primaria. A juicio de los disidentes la norma en cuestión altera este sistema de prohibiciones estipulado constitucionalmente.

$\mathrm{Al}$ efecto se señala:

"8. Que para analizar si este agregado que hace la norma en análisis es legitimo desde el punto de vista constitucional, hay que considerar ciertas variables del sistema de primarias que el proyecto define. En primer lugar, participar o no en las primarias en un acto voluntario, tanto para los partidos como para los candidatos (articulos $1^{\circ}$ y $2^{\circ}$ ). En segundo lugar, las primarias son simultáneas para todos los candidatos de los partidos y pactos que participen en ellas. Es decir, se realizan de manera conjunta en todo el pais (artículo $3^{\circ}$ ). En tercer lugar, la elección primaria es una votación popular. Asi lo estableció esta Magistratura en la STC 279/1998; y asi lo consagra el propio proyecto, al distinguir, en su articulo 46, dos tipos de elecciones populares: la primaria y la definitiva. Eso explica muchos de los elementos del sistema de elecciones primarias, como que intervengan organismos públicos en su organización (el Servicio Electoral y el Tribunal Calificador de Elecciones) (artículos $3^{\circ}$ y 31), asi como que se establezca la existencia de vocales con función obligatoria (articulo 27), etc.; 
9. Que, asi las cosas, consideramos que el agregado que el articulo hace a la norma constitucional, la vulnera, en primer lugar, porque relativiza la inhabilidad. Mientras la Constitución establece que no pueden ser candidatos, en esa elección, al respectivo cargo, la ley agrega que esa probibición es solamente aplicable en el territorio electoral en que el candidato participó en primaria. Es decir, la norma permite que quien perdió en una elección primaria en una determinada circunscripción, distrito o comuna, pueda ir en la elección definitiva como candidato, si lo hace en otro territorio electoral.

En segundo lugar, afecta la seriedad del proceso, al que se ingresa voluntariamente, utilizando bienes y recursos del Estado. Si la Constitución establece que no pueden ser candidatos los perdedores, la ley no puede abrir una puerta para que si puedan hacerlo por otra vía.

El respeto hacia la inhabilidad de los perdedores es uno de los pilares del sistema de elecciones primarias. No puede permitirse que el legislador perfore dicho sistema, estableciendo una variable que la Constitución no contempla, pues ello equivaldría a su reforma sin cumplir el quórum requerido al efecto;"

La Ministra Marisol Peña Torres y el Ministro José Antonio Viera-Gallo, estuvieron por declarar que los artículos 44 y 45 del proyecto, que remiten a los ilícitos penales establecidos en la Ley No 18.700, es constitucional, puesto que a su juicio se cumple con el estándar de especificidad necesario en los tipos penales, y que las expresiones en "lo que fuere procedente" o "en lo que corresponda", simplemente tienen el objetivo de precisar la remisión:

"1 ${ }^{\circ}$. Que las oraciones "Los actos en contravención a esta ley se sancionarán de conformidad a lo señalado en el Título VII de la Ley $N^{\circ} 18.700$, en lo que fuere procedente" y "A los partidos políticos que no den cumplimiento a lo establecido en esta ley, o en sus estatutos, respecto de la realización de elecciones primarias, les serán aplicables las disposiciones contenidas en los Título VIII y IX de la Ley $N^{\circ} 18.603$, en lo que corresponda", contenidas en los artículos 44 y 45 del proyecto de ley examinado, no carecen de la tipicidad necesaria para cumplir el estándar constitucional. En efecto, de la lectura de los preceptos contenidos en el proyecto de ley de que se trata se desprenden los distintos deberes que se asignan a los partidos políticos que opten por realizar elecciones primarias para la nominación de los candidatos a los cargos de Presidente de la República, de parlamentarios y de alcaldes, por lo que queda claro que es la infracción de esos deberes la que será conocida, en su caso, por un miembro del Tribunal Calificador de Elecciones designado por sorteo, por aplicación del articulo 56 de la Ley $N^{\circ} 18.603$, a la que se remite expresamente el artículo 45 que se comenta. En lo que se refiere al artículo 44, por su parte, es clara, asimismo, la remisión al Título VII de la Ley $N^{\circ} 18.700$ que establece las diferentes sanciones para quienes intervengan en los actos electorales incumpliendo los deberes inherentes a su actuación;

$2^{\circ}$. Que las expresiones "en lo que fuere procedente" y "en lo que corresponda", incluidas en los artículos 44 y 45 aludidos, sólo tienen por objeto clarificar que la remisión a las normas de las leyes $N^{\circ} 18.700$ y 18.603 se efectuará teniendo presente las particularidades de las elecciones primarias en forma concordante con lo dispuesto en el artículo $6^{\circ}$ del mismo proyecto de ley consultado, lo que no merece a estos disidentes objeciones de constitucionalidad; 
$3^{\circ}$. Que, a mayor abundamiento, quienes suscriben este voto consideran que las remisiones normativas que efectúan los artículos 44 y 45 del proyecto de ley consultado se realizan a otras normas con el mismo rango de ley-leyes $N^{\circ}$ s 18.700 y 18.603-, tanto para la determinación de las penas correspondientes como del tribunal y del procedimiento aplicable para imponerlas, lo que no infringe la Carta Fundamental, en la medida que el principio de legalidad que debe imperar en materia penal se encuentra bien resguardado,"

Los Ministros Carlos Carmona Santander y Gonzalo García Pino, estuvieron por declarar la inconstitucionalidad del Art. 6º del proyecto, que establece la aplicación supletoria de la Ley № 18.700, por cuanto la Constitución encomienda a la ley orgánica constitucional la regulación de un sistema de elecciones primarias, y no siendo estas iguales a las definitivas, la remisión a las normas de la Ley No 18.700, no cumple con lo mandatado por el Art. 19 № 15 de la Constitución:

"9. Que, como se observa, la disposición establece el régimen jurídico que rige a las elecciones primarias, pues establece cuáles son las normas supletorias de lo que el proyecto no regula expresamente;

10. Que mientras en una serie de disposiciones el mismo proyecto va remitiendo para incluir o excluir a ciertas disposiciones especificas de dichos cuerpos normativos (por ejemplo, respecto de la Ley $N^{\circ}$ 18.700, los artículos 13, 15, 17, 18, 19, 26, 29, 30, 33, 36, 39 y 44 remiten a normas precisas de ella. En relación a la Ley $N^{\circ} 18.695$, hay remisiones expresas en los artículos 13, 18, 36 y 39. Respecto de la Ley $N^{\circ} 19.884$, hay remisiones expresas en los artículos 41 y 43), en la disposición que se analiza la remisión es genérica, pues se aplican dichos cuerpos normativos "en todo lo que no sea contrario a ella y en lo que le sea aplicable";

11. Que consideramos que dicha remisión no se ajusta a la Constitución. En primer lugar, porque no cumple el mandato del articulo 19, $N^{\circ} 15$, de la misma Carta Fundamental, que obliga a la ley orgánica a establecer un sistema de elecciones primarias. Ello exige que el marco jurídico que lo regule no pueda generar incertidumbre. No puede haber un marco claro y preciso si no se conoce con precisión qué preceptos de las leyes remitidas lo regularán o quién va a decidir qué precepto de estas leyes no es contrario o le resulta aplicable.

En segundo lugar, cuando la Constitución establece una ley orgánica, define las materias que puede abordar. Esta definición puede ser abierta o cerrada. Pero el legislador debe abordar la regulación de esas materias en lo medular. De lo contrario, no cumple el encargo de la Constitución de complementar sus mandatos de manera armónica y sistemática;

12. Que, por lo antes expuesto, consideramos que el artículo $6^{\circ}$ del proyecto es inconstitucional."

Finalmente, los Ministros Gonzalo García Pino y Carlos Carmona Santander, estuvieron por declarar la inconstitucionalidad del Art. 43 del proyecto, que señala que el candidato deberá presentar una declaración jurada del gasto realizado a la campaña primaria, por cuanto se puede utilizar hasta un diez por ciento del límite de gasto de campaña en la elección primaria. 
A juicio de los disidentes, esta norma va en contra del sistema de regulación de gastos de la Ley No 19.884, el que se basa en un control de gastos realmente efectuados, cuestión que no puede realizarse en base a una declaración jurada, vulnerando el sistema de control de gastos que manda a establece la Constitución, en su Art. 18.

"5. Que la disposición es inconstitucional, en primer lugar, porque excluye la regla general de gasto electoral de la Ley $N^{\circ} 19.884$ en materia de rendición de cuentas. Se crea, por tanto, un régimen privilegiado;

6. Que, en segundo lugar, la norma afecta el artículo 18 de la Carta Fundamental, en cuanto se debe garantizar siempre "la plena igualdad entre los independientes y los miembros de partidos politicos tanto en la presentación de candidaturas como en su participación en los señalados procesos". En efecto, un independiente y un candidato de partidos o pactos electorales que no hayan participado en primarias, tiene un limite de gasto definido en el 100\% de los valores que establece la Ley $N^{\circ} 19.884$. En cambio, un candidato nominado en primarias tendrá un límite real de un $90 \%$ de autorización máxima de gasto. Esta diferencia justificada se diluye si es que la rendición de gasto depende sólo de la voluntad del candidato seleccionado en primarias afectando, con ello, la justicia de las reglas de competencia en igualdad de condiciones entre los respectivos candidatos en la elección definitiva;

\section{(...)}

8. Que, en tercer lugar, se vulnera el articulo 18 de la Constitución, que establece "un sistema de financiamiento, transparencia, limite y control del gasto electoral". El constituyente, en la ley de reforma constitucional $N^{\circ} 20.337$, dispuso, al acentuar el concepto de transparencia, que el mandato legislativo fuera amplio pero respetuoso de esa condición de transparencia y exigiendo "su control". Entonces, el constituyente enmarca la interpositio legislatoris con el deber de respetar su contenido minimo. Y cuando una norma permite total opacidad, impidiendo el control de transparencia, no puede sino ser contraria a lo que la Constitución manda;

9. Que, por todas estas razones, consideramos que la regla del articulo 43, que permite una declaración de cuentas vía una declaración jurada, es inconstitucional."

\section{Conclusión}

La sentencia comentada retorna a viejos temas de la jurisprudencia del Tribunal Constitucional y que resultan congruentes con su doctrina; a saber: ley orgánica constitucional y su dominio, legislación secundaria y límites de la potestad normativa confiada a servicios públicos, limitación de derechos políticos y alcances de los principios de legalidad y tipicidad penal.

También resultan interesantes las numerosas disidencias contenidas en la sentencia sobre aspectos medulares en torno a la declaración de inconstitucionalidad (v. gr. Potestad normativa del Servicio Electoral, derechos e identificación del ciudadano elector, y admisión de la remisión abierta en la ley penal. 
Nos interesa en este lugar abordar dos temas sinópticamente: ley orgánica constitucional y potestad normativa sub lege. En relación a la ley orgánica constitucional objeto de control preventivo, abstracto y obligatorio, el Tribunal admitiendo el criterio de las cámaras en el iter legis, destaca la amplitud del dominio del legislador orgánico: el "sistema de elecciones primarias", que además se extiende a otras normas en razón de las múltiples reservas al legislador orgánico existente en nuestro derecho electoral. Al mismo tiempo reitera el Tribunal su doctrina que las leyes orgánicas constitucionales son jerárquicamente leyes, revestidas de una "superlegalidad formal" para cautelar su estabilidad normativa derivada de los quórums agravados en la lógica del principio de competencia. A su vez en relación a la potestad normativa de servicios públicos como el Servicio Electoral, el Tribunal reitera su doctrina en orden a que el principio de legalidad y jerarquía normativa se impone por partida doble: primero la ley debe ser la fuente expresa de la potestad normativa y segundo la potestad normativa secundaria es sub Constitutione y sub lege; y en tal medida las resoluciones que se adopten sólo podrán reglamentar aspectos de detalle o pormenorizar las previsiones legislativas lo obvio es también admitir que la ley orgánica constitucional admite desarrollo infralegal: reglamentario o sub reglamentario.

Con todo, observamos dos inconsistencias en la doctrina del Tribunal Constitucional.

Primero homologar de alguna manera la potestad normativa de servicios públicos en general con la potestad normativa de entes públicos de la Administración del Estado dotados de autonomía constitucional, en que también dicha autonomía se proyecta competencialmente a su potestad normativa, y lo propio en menor intensidad tratándose de autonomías legales como las superintendencias; que cierta doctrina encuadra en el modelo de "administraciones independientes". Ocurre aquí que la potestad normativa secundaria no debe ser examinada sólo a la luz del principio de jerarquía normativa sino debe ser corregido dicho principio ordenador de las fuentes conforme al principio de competencia.

Segundo, de vueltas el Tribunal al dominio del legislador orgánico da recibo a un concepto abierto o extendido de la competencia de este tipo de ley, sea vía hermenéutica al definir el concepto de "sistema", sea al dar recibo a otras reservas al legislador orgánico en el derecho electoral. Tal doctrina del Tribunal Constitucional contrasta con una práctica consolidada de examinar con estricto apego a la idea de excepcionalidad del dominio del legislador orgánico aquellas materias determinadas por las cámaras de quórum especial. La inconsistencia es plena al dar recibo a la noción de "superlegalidad", cuando la legislación de supermayorías, sólo cautela la estabilidad normativa a través de quórums reforzados, con la finalidad contramayoritaria de forzar el consenso en la dictación y reforma de la ley orgánica constitucional; distorsionando así el principio democrático. 